SISSA $16 / 98 / \mathrm{FM}$

KCL-MTH-98-06

CTP TAMU-5/98

hep-th/9803041

\title{
Open Superbranes
}

\author{
C.S. $\mathrm{Chu}^{1}$, P.S. Howe ${ }^{2}$, E. Sezgin ${ }^{3 \dagger}$ and P.C. West $^{2}$ \\ 1 International School for Advanced Studies (SISSA), Via Beirut 2, 34014 Trieste, Italy \\ 2 Department of Mathematics, King's College, London, UK \\ 3 Center for Theoretical Physics, Texas A\&M University, College Station, TX 77843, USA
}

\begin{abstract}
Open branes ending on other branes, which may be referred to as the host branes, are studied in the superembedding formalism. The open brane, host brane and the target space in which they are both embedded are all taken to be supermanifolds. It is shown that the superspace constraints satisfied by the open brane are sufficient to determine the corresponding superspace constraints for the host branes, whose dynamics are determined by these constraints. As a byproduct, one also obtains information about the boundary of the open brane propagating in the host brane.
\end{abstract}

\footnotetext{
${ }^{\dagger}$ Research supported in part by NSF Grant PHY-9722090
} 


\section{Introduction}

It is well-known that certain open branes can end on other branes provided that the dimensionalities of the branes are chosen correctly [1-16]. These configurations are intimately connected with the special cases of intersecting branes where the dimension of the intersection manifold coincides with one of the intersecting brane boundary dimension. This is known to occur for fundamental string or $\mathrm{D}(p-2)$-branes ending on $\mathrm{D} p$-branes and $\mathrm{D} p$-branes ending on NS 5 -branes $(1 \leq p \leq 6)$. (See [13] and [14] for an extensive analysis).

Recently, such systems have been discussed in a hybrid Green-Schwarz formalism in M-theory and in the context of D-branes in ten dimensions [15, 16]. (For earlier related work, see [8, 9, 10, 11]). The basic idea of references [15] and [16] is to write down the Green-Schwarz (GS)action for a brane, for example the M2-brane, with a boundary. The worldvolume for the GS action is a bosonic manifold $\Sigma$ while the target space in this case is the superspace for elevendimensional supergravity, $\underline{M}$. The boundary of the worldvolume, $\partial \Sigma$, is taken to be embedded in a supermanifold $M$ which is also embedded in $\underline{M}$. The supermanifold $M$ is taken to be the worldsurface of the M5-brane, and it was found that $\kappa$-symmetry of the M2-brane action with boundary contained in $M$ imply the equations of motion for the M5-brane. These results were extended to strings and $\mathrm{D}(p-2)$ branes ending on $\mathrm{D} p$-branes in [16]. The formalism used in these papers is therefore a hybrid one, since the first brane is treated from the GS point of view while the second one is treated using the superembedding formalism. Since $\kappa$-symmetry is a relic of local worldsurface supersymmetry in the superembedding formalism it is to be expected that the same results can be obtained by working entirely in the superembedding formalism. This is indeed the case as we shall show in the current paper. We shall again focus on M-branes and D-branes.

In the approach presented here, it is not necessary to exhibit the open-host brane system as a classical solution of the target space theory, nor is it necessary to make assumption on the topology of the host brane. For example, the host brane itself may be closed or open. An open host brane, can in turn end on another suitable secondary host brane and so on. Thus one can obtain a brain chain. A special case of this arises when all members of the chain have the same dimension, thus forming a brane network. The two possibilities allowed are those which use the string ending on D1-brane junction as building block [17, 18, 19], and those which use D5-branes ending on NS 5-branes as building blocks [20]. Here, for the purposes of the present paper, we shall focus on the basic building blocks of chain or network configurations, namely an open brane ending on a host brane.

The next three sections are devoted to the discussion of an M2-brane ending on an M5-brane, fundamental strings ending on $\mathrm{D} p$-branes and $\mathrm{D} p$-branes ending on $\mathrm{D}(p+2)$-branes, respectively. A summary of our results and further comments about them are provided in the Conclusions.

\section{Open branes in M-theory}

We consider the following picture: a 2-brane worldsurface $M_{2}$, with (even|odd) dimension (3|16) can end on an M5-brane worldsurface $M_{5}$ (dimension (6|16)), via a boundary $M_{1}$ which is a supermanifold of dimension (2|8). Thus $M_{1}=\partial M_{2}$ and $M_{1} \subset M_{5}$ while both $M_{2}$ and $M_{5}$ are embedded in an (11|32)-dimensional target space $\underline{M}$. We therefore have embeddings 


$$
f_{i}: M_{i} \hookrightarrow \underline{M} ; \quad i=1,2,5
$$

as well as an embedding

$$
f_{1}^{5}: M_{1} \hookrightarrow M_{5}
$$

Clearly

$$
f_{1}=f_{5} \circ f_{1}^{5}
$$

The fact that the 2-brane can end on a supermanifold which has bosonic dimension two is related to the fact that the 5-brane admits stringlike soliton solutions to its equations of motion [21]. We shall comment on the possibility of host branes other than the M5-brane in the Conclusions.

The boundary of the open membrane, in general, may consist of an arbitrary number of closed strings. However, to keep the discussion as simple as possible, we shall consider an open membrane that has the topology of a disk, and hence a single component boundary, a closed string. Our analysis can easily be extended for multi-component boundaries, with essentially same results.

We shall now demonstrate that if the standard embedding condition is assumed for the 2-brane then the standard embedding condition for the 5-brane is implied. We shall also show, although it is not essential for the derivation of the M5-brane equations of motion, that this picture requires for its consistency a 2 -form gauge potential $A$ on $M_{5}$ whose (modified) 3 -form field strength $\mathcal{F}$ is only non-vanishing when all of its indices are bosonic. This is not essential for the derivations of the M5-brane equations of motion because, as has been shown elsewhere [22], this result actually follows from the embedding condition. However, it is useful to introduce this discussion here as it will play a more significant rôle in the analysis of D-branes.

To make the analysis of the embedding conditions we introduce the embedding matrices $E$ which are simply the derivatives of the embeddings given above referred to standard bases. Thus we have the following set of embedding matrices,

$$
E_{A_{1}} \underline{A}, E_{A_{2}} \underline{A}, E_{A_{5}} \underline{A} \text { and } E_{A_{1}}^{A_{5}}
$$

corresponding to the derivatives of the embeddings $f_{1}, f_{2}, f_{5}$, and $f_{1}{ }^{5}$ respectively. The notation here is that underlined indices refer to the target space $\underline{M}$ while indices for each of the manifolds $M_{i}, i=1,2,5$ are distinguished by appending to them the corresponding numerical subscripts. As usual, indices from the beginning of the alphabet are preferred basis indices while indices from the middle of the alphabet denote coordinate indices. Capital indices run over both bosonic and fermionic indices while latin (greek) letters are used for bosonic (fermionic) indices separately, for example $A=(a, \alpha)$. We shall denote normal indices by primes; it should be clear from the context which embedding is being referred to when a normal index is employed. A more explict formula for the embedding matrix is, using the 2-brane as an example,

$$
E_{A_{2}} \underline{A}=E_{A_{2}}^{M_{2}} \partial_{M_{2}} z^{\underline{M}} E_{\underline{M}}^{\underline{A}},
$$


where we have introduced the vielbein matrices, $E_{\underline{M}} \underline{A}$ etc., which relate the preferred bases to the cooordinate bases.

The basic embedding condition for the 2-brane is

$$
E_{\alpha_{2}} \underline{a}=0
$$

This equation simply states that the odd tangent space to $M_{2}$ is a subspace of the odd tangent space to $\underline{M}$ at each point in $M_{2} \subset \underline{M}$. It can be shown that this condition determines the equations of motion of the membrane and also that the geometry of the target space is required to be that of on-shell eleven-dimensional supergravity. On the boundary $M_{1}=\partial M_{2}$ we therefore have

$$
E_{\alpha_{1}} \underline{a}=0
$$

By the chain rule, we have

$$
E_{A_{1}} \underline{A}=E_{A_{1}}^{A_{5}} E_{A_{5}} \underline{A}
$$

and so

$$
0=E_{\alpha_{1}} \underline{a}=E_{\alpha_{1}}^{\alpha_{5}} E_{\alpha_{5}} \underline{a}+E_{\alpha_{1}}^{a_{5}} E_{a_{5}} \underline{a} .
$$

We now introduce a complementary normal matrix in the bosonic sector for $M_{5}$ in $\underline{M}$ denoted by $E_{a_{5}^{\prime}} \underline{a}$. The inverse of the pair $\left(E_{a_{5}} \underline{a}, E_{a_{5}^{\prime}} \underline{a}\right)$ is denoted by $\left(\left(E^{-1}\right)_{\underline{a}}^{a_{5}},\left(E^{-1}\right)_{\underline{a}} a_{5}^{\prime}\right)$. Multiplying (9) by $\left(E^{-1}\right)_{\underline{a}}{ }^{\prime}$ we find

$$
E_{\alpha_{1}}^{\alpha_{5}} E_{\alpha_{5}} \underline{a}_{\left(E^{-1}\right)_{\underline{a}}^{a_{5}^{\prime}}=0}
$$

while multiplying the same equation by $\left(E^{-1}\right)_{\underline{a}}{ }^{a_{5}}$ we get

$$
E_{\alpha_{1}}{ }^{\alpha_{5}} E_{\alpha_{5}}{ }^{\underline{a}}\left(E^{-1}\right)_{\underline{a}}^{a_{5}}+E_{\alpha_{1}}^{a_{5}}=0 \text {. }
$$

Now for any superembedding it is always possible to choose the odd tangent space of the embedded submanifold such that (taking $M_{5} \subset \underline{M}$ as an example)

$$
E_{\alpha_{5}} \underline{a}^{\left(E^{-1}\right)_{\underline{a}}^{a_{5}}=0}
$$

To see this we observe that the odd tangent space basis $E_{\alpha_{5}}$ for $M_{5}$ can be written quite generally as

$$
E_{\alpha_{5}}=E_{\alpha_{5}} \underline{\alpha} E_{\underline{\alpha}}+E_{\alpha_{5}} \underline{a} E_{\underline{a}}
$$

while for the even subspace we can write

$$
E_{a_{5}}=E_{a_{5}} \underline{\alpha} E_{\underline{\alpha}}+E_{a_{5}} \underline{a} E_{\underline{a}}
$$


Hence if we redefine $E_{\alpha_{5}}$ by

$$
E_{\alpha_{5}} \rightarrow \tilde{E}_{\alpha_{5}}=E_{\alpha_{5}}+\Lambda_{\alpha_{5}}^{a_{5}} E_{a_{5}}
$$

for some fermionic superfield $\Lambda_{\alpha_{5}}{ }^{a_{5}}$ we find

$$
\tilde{E}_{\alpha_{5}} \underline{\underline{a}}=E_{\alpha_{5}} \underline{\underline{a}}+\Lambda_{\alpha_{5}}^{a_{5}} E_{a_{5}} \underline{a} .
$$

Multiplying this equation with $\left(E^{-1}\right)_{\underline{a}}{ }^{a_{5}}$, we observe that the quantity $E_{\alpha_{5}} \underline{a}\left(E^{-1}\right)_{\underline{a}}^{a_{5}}$ can always be made to vanish by choosing $\Lambda_{\alpha_{5}}{ }^{a_{5}}$ aprropriately. Thus, the result (12) is proved. Using this result in conjunction with (10), $M_{1}$ being arbitrary, we see that the odd tangent space of $M_{5}$ can always be chosen such that

$$
E_{\alpha_{5}} \underline{a}=0
$$

and this implies, from (11), that

$$
E_{\alpha_{1}}^{a_{5}}=0
$$

as well.

Equation (17) is the standard embedding condition for the M5-brane. It has been shown that this equation determines the equations of motion for the 5-brane [22, 23]. Furthermore, the boundary brane $M_{1}$ also obeys the standard embedding condition as a subsupermanifold of $M_{5}$. We therefore conclude that the 5-brane equations of motion are implied by requiring the consistency of 2-branes ending on 5-branes.

The embedding constraint (18) for the boundary string confined to propagate within the 5-brane is rather interesting. We shall comment further on this point in the Conclusions, but here we shall focus on the derivation of the 5-brane equations of motion.

Let us now consider the Wess-Zumino form $W_{4}$ for the 2-brane. As this brane is type I, i.e. its worldsurface multipet contains only scalars as bosonic degrees of freedom, $W_{4}$ is simply the pull-back of the target-space 4-form field strength of eleven-dimensional supergravity

$$
G_{4}=d C_{3} .
$$

Since $W_{4}$ is a closed form on a supermanifold with bosonic dimension three, it must be exact because the de Rham cohomology of a supermanifold coincides with that of its body. Thus we can write [24, 25, 26]

$$
W_{4}=f_{2}^{*} G_{4}=d K_{3}
$$

for some globally defined 3-form $K_{3}$ on $M_{2}$. We therefore have

$$
K_{3}=d Y_{2}+f_{2}^{*} C_{3} \quad \text { on } M_{2} \text {. }
$$


Since both of the potentials $Y_{2}$ and $C_{3}$ are, in general, locally defined, the fact that $K_{3}$ is globally defined dictates the transformation rule for $Y_{2}$. On the boundary $M_{1}$ we identify $Y_{2}$ as the pull-back of a 2-form potential on $M_{5}$,

$$
Y_{2}=\left(f_{1}^{5}\right)^{*} A_{2} \quad \text { on } M_{1}
$$

This implies that the corresponding field strength 3-form on $M_{5}$ should be defined by

$$
\mathcal{F}_{3}=d A_{2}+f_{5}^{*} C_{3} \quad \text { on } M_{5}
$$

The associated Bianchi identity is

$$
d \mathcal{F}_{3}=f_{5}^{*} G_{4}
$$

It is straightforward to demonstrate that the only non-vanishing component of $K_{3}$ is the one with purely bosonic indices, so that it must vanish when restricted to the boundary $M_{1}$. This implies in turn that $\mathcal{F}_{3}$ must vanish on $M_{1} \subset M_{5}$. The pull-back of $\mathcal{F}_{3}$ to $M_{1}$ is given by

$$
\left(f_{1}^{5}\right)^{*} \mathcal{F}_{A_{1} B_{1} C_{1}}=E_{C_{1}}{ }^{C_{5}} E_{B_{1}}{ }^{B_{5}} E_{A_{1}}^{A_{5}} \mathcal{F}_{A_{5} B_{5} C_{5}},
$$

up to Grassmann sign factors which we suppress. Since the left-hand side vanishes, and since $E_{\alpha_{1}}{ }^{a_{5}}=0$, we conclude, $M_{1}$ being arbitrary, that this can only be satisfied if

$$
\mathcal{F}_{\alpha_{5} B_{5} C_{5}}=0
$$

that is, $\mathcal{F}_{3}$ must be purely even on $M_{5}$.

To summarise then, we have shown that the consistency of the picture of a 2-brane with a boundary ending on a 5-brane in which the boundary is embedded requires that, if the standard embedding condition (6) for the 2-brane is imposed, the standard embedding condition for the 5-brane should also hold and that there is a 2-form gauge potential on $M_{5}$ whose 3 -form field strength should satisfy equation (26) above. This is in perfect agreement with the results of [15] obtained from a hybrid approach mentioned in the introduction. As we have remarked earlier, the second of these equations actually follows from the first in the case of M-branes, but this is not true for all D-branes [27].

\section{Fundamental strings ending on D-branes}

The discussion of the previous section can be carried over straightforwardly to fundamental type II strings in ten dimensions ending on D-branes. In general, the end points of the open string may lie on two different $\mathrm{D} p$-branes or one end-point of a semi-infinite open string may lie on a $\mathrm{D} p$-brane while the other end is feely moving. It is sufficent to consider the case where both endpoints are ending on a single $\mathrm{D} p$-brane for the purpose of deriving the constraints that govern the dynamics of the $\mathrm{D} p$-brane. It is straightforward to generalize the discussion for the other two cases. Thus we have the following supermanifolds: the string manifold, $M_{1}$ (dimension (2|16)), its boundary $M_{0}=\partial M_{1}$ (dimension (1|8)), the worldvolume of the $\mathrm{D} p$-brane $M_{p}$ (dimension 
$(p+1 \mid 16))$ and the target space $\underline{M}$ which is either type IIA or type IIB superspace and which has dimension $(10 \mid 32)$. The associated embeddings are

$$
f_{i}: M_{i} \hookrightarrow \underline{M}, \quad i=0,1, p
$$

and

$$
f_{0}^{p}: M_{0} \hookrightarrow M_{p}
$$

with

$$
f_{0}=f_{p} \circ f_{0}^{p}
$$

For either of the fundamental strings the embedding condition

$$
E_{\alpha_{1}} \underline{a}=0
$$

implies the equations of motion for the string. By using exactly the same procedure as in the previous section we conclude that the embedding condition will also hold for the $\mathrm{D} p$-brane on which it ends, so that

$$
E_{\alpha_{p}} \underline{a}=0
$$

In addition the standard embedding condition will also hold for the worldvolume of the 0-brane boundary considered as a subsupermanifold of $M_{p}$ :

$$
E_{\alpha_{0}}{ }^{a_{p}}=0
$$

Each string has a Wess-Zumino 3-form $W_{3}=d Z_{2}$ which is simply the pull-back of the NS 3-form

$$
H_{3}=d B_{2}
$$

on the target space, so we have

$$
W_{3}=f_{1}^{*} H_{3}
$$

The Wess-Zumino form is exact on $M_{1}$ so

$$
W_{3}=d K_{2}
$$

from which we conclude that

$$
K_{2}=d Y_{1}+f_{1}^{*} B_{2} \quad \text { on } M_{1}
$$

On the boundary we identify $Y_{1}$ with the pull-back of a 1-form gauge potential $A_{1}$ on $M_{p}$ : 


$$
Y_{1}=\left(f_{1}^{p}\right)^{*} A_{1} \quad \text { on } M_{0} \text {. }
$$

We can thus identify the modified field strength 2 -form $\mathcal{F}_{2}$ on $M_{p}$ as

$$
\mathcal{F}_{2}=d A_{1}+f_{p}^{*} B_{2} \quad \text { on } M_{p},
$$

with the associated Bianchi identity

$$
d \mathcal{F}_{2}=f_{p}^{*} H_{3} .
$$

It follows from the constraints on the fundamental string that the only non-vanishing component of the two-form $K_{2}$ is the one with purely bosonic indices and so it vanishes on the boundary. By a similar argument to that given in the preceeding section, we therefore conclude that

$$
\mathcal{F}_{\alpha_{p} B_{p}}=0
$$

The embedding condition (31) and the $\mathcal{F}$-constraint (40) are together sufficient to imply the equations of motion for the $\mathrm{D} p$-brane in all cases. They are not necessarily necessary, however. In type IIA one can have $p=2,4,6,8$. For $p=2,4$, the embedding condition is already enough to imply the equations of motion while for $p=6,8$, the $\mathcal{F}$-constraint is required as well. It is clear that an additional constraint is required for $p=8$ since the brane has co-dimension one. In this case the worldvolume multiplet determined by the embedding condition is an entire scalar superfield. That an additional constraint is required in the case of $p=6$ is less obvious since the worldvolume multiplet is in this case a $d=7$ "linear multiplet", i.e. a superfield whose leading component is three scalars and whose next leading component is a spin-half field in seven dimensions. There is also a 0-brane in type IIA for which the embedding condition alone is sufficient to give the dynamics. In type IIB there are $D$-branes for all odd $p$. For $p=1,3,5$ the embedding condition gives the equations of motion while for $p=7,9$ the $\mathcal{F}$-constraint is required as well. For $p=7$ the worldvolume multiplet determined by the embedding condition is a chiral scalar superfield, which is otherwise unconstrained, and for $p=9$ there are no scalars so that it is clear that an additional constraint is required. Further details of $D$-brane embeddings will be found elsewhere [27]. (The case of D9-brane has been recently treated from the superembedding point of view in [28]).

\section{D-branes ending on D-branes}

In this section we shall consider $\mathrm{D} p$-branes ending on $\mathrm{D}(p+2)$-branes of the Type IIA/B superstring theories in ten dimensions. The discussion is very similar to the preceding two cases. It will enable us to recover the results of [16] in a superspace approach.

The relevant supermanifolds are: the $\mathrm{D} p$-brane manifold, $M_{p}$ (dimension $(p+1 \mid 16)$ ), its boundary $M_{p-1}=\partial M_{p}$ (dimension $\left.(p \mid 8)\right)$, the worldvolume of the $\mathrm{D}(p+2)$-brane $M_{p+2}$ (dimension $(p+$ $3 \mid 16)$ ) and the target space $\underline{M}$ which is either type IIA or type IIB superspace and which again has dimension (10|32). The associated embeddings are

$$
f_{i}: M_{i} \hookrightarrow \underline{M}, \quad i=p-1, p, p+2
$$


and

$$
f_{p-1}^{p+2}: M_{p-1} \hookrightarrow M_{p+2}
$$

with

$$
f_{p-1}=f_{p+1} \circ f_{p-1}^{p+2} .
$$

For the $\mathrm{D} p$-brane the embedding condition

$$
E_{\alpha_{p}} \underline{a}=0
$$

is assumed to hold, as well as the $\mathcal{F}$-constraint

$$
\mathcal{F}_{\alpha_{p} B_{p}}=0 \text {. }
$$

Using the same argument as before we deduce that the standard embedding condition

$$
E_{\alpha_{p+2}} \underline{a}=0
$$

will hold for the $\mathrm{D}(p+2)$ brane as well. In addition the standard embedding condition will also hold for the worldvolume of the $(p-1)$-brane boundary considered as a subsupermanifold of $M_{p+2}$ :

$$
E_{\alpha_{p-1}}^{\alpha_{p+2}}=0 .
$$

The Wess-Zumino form for a $\mathrm{D} p$-brane is $W_{p+2}=d Z_{p+1}$ where the Wess-Zumino potential is given as the $p+1$-form component of an inhomogeneous potential form

$$
Z_{p+1}=\left(f_{p}^{*} C e^{\mathcal{F}}\right)_{p+1},
$$

where $C$ is the sum of the RR potential forms on the target space. In the case of massive type IIA D $p$-branes, the term $m f_{p}^{*} \omega_{p+1}$, where $m$ is the mass parameter and $\omega_{p+1}(A, d A)$ is the Chern-Simons form, has to be added to the right hand side [29].

$W_{p+2}$ is a closed form on $M_{p}$ and by the same arguments that were used before it must be exact, so that we can write

$$
W_{p+2}=d K_{p+1}
$$

for some globally defined $(p+1)$-form $K_{p+1}$ on $M_{p}$. Clearly

$$
K_{p+1}=d Y_{p}+Z_{p+1} \quad \text { on } M_{p}
$$

On the boundary we identify $Y_{p}$ with the pull-back of a $p$-form potential $A_{p}$ on $M_{p+2}$ : 


$$
Y_{p}=\left(f_{p-1}^{p+2}\right)^{*} A_{p} \quad \text { on } M_{p-1}
$$

We also identify the 1-form potential of the $\mathrm{D} p$-brane on the boundary with the pull-back of the 1-form potential of the $\mathrm{D}(p+2)$-brane and use the same letter $A_{1}$. The field strength $(p+1)$-form associated with $A_{p}$ is

$$
\mathcal{F}_{p+1}=d A_{p}+f_{p+2}^{*}\left(C e^{\mathcal{F}}\right)_{p+1} \quad \text { on } M_{p+2},
$$

which obeys the Biachi identity

$$
d \mathcal{F}_{p+1}=\left(G e^{\mathcal{F}}\right)_{p+2}
$$

with the definition

$$
G=d C-C H
$$

In the case of massive type IIA D $p$-branes, the Chern-Simons term $m \omega_{p+1}$ needs to be added to the right hand side of this definition but the same Bianchi identity (53) holds.

The equations for the $p$-brane imply that the only non-vanishing component of $K_{p+1}$ is the one with purely bosonic indices and so we deduce that the pull-back of $\mathcal{F}_{p+1}$ to $M_{p-1}$ must vanish and this implies the $\mathcal{F}$-constraint for $\mathcal{F}_{p+1}$, namely

$$
\mathcal{F}_{\alpha_{p+2} B_{p+2} C_{p+2} \ldots}=0 \text {. }
$$

The equations for the $\mathrm{D}(p+2)$-brane derived from letting a $\mathrm{D} p$-brane end on it are therefore the standard embedding condition (46) together with $\mathcal{F}$-constraints of the form of equation (55) for both a 2 -form field strength $\mathcal{F}_{2}$ and a $(p+1)$-form field strength $\mathcal{F}_{p+1}$. These field strengths are essentially duals of one another. To be more precise, at the linearised level $\mathcal{F}_{a b}$ is the dual of $\mathcal{F}_{a_{1} \ldots a_{p+1}}$, but in the full theory there are non-linear corrections [16].

\section{Conclusions}

In this paper we have seen yet again the power of superembeddings in the description of superbrane dynamics. Starting from simple geometrical considerations having to do with the way an open M2-brane is embedded in eleven dimensions or the way an open string or $\mathrm{D} p$-brane is embedded in ten dimensions, we were able to derive the superfield constraints that govern the dynamics of the host branes on which these open branes end. The constraints consist of the embedding condition of the host brane and a constraint on a suitable field strength living on the host brane, namely $\mathcal{F}_{3}$ for the M5-brane, $\mathcal{F}_{2}$ for the $\mathrm{D} p$-brane and $\mathcal{F}_{p+1}$ for the $\mathrm{D}(p+2)$ branes. The first two case are the most familiar ones while the last case is somewhat novel in that it contains a $p$-form potential $A_{p}$ as well as the usual Maxwell field $A_{1}$, in accordance with the results of [16]. As mentioned earlier, these are dual to each other (in a highly nonlinear fashion) and consequently we expect to acquire new insights about duality symmetries within this framework. 
As a byproduct of our approach to open superbranes, we have obtained the boundary embedding conditions (18), (32) and (47) that characterize the embedding of the boundary manifolds within the host branes, e.g. the closed string boundary of the M2-brane within the host M5-brane, as given in (18). These constraints are not sufficient, however, to put the boundary brane on-shell.

Consider, for example, the case of $\mathrm{D} p$-branes ending on $\mathrm{D}(p+2)$-branes, where there are boundary $(p-1)$-branes embedded in $(p+2)$-branes. Note that $1 \leq p \leq 7$. In the special case of the D7-brane ending on the D9-brane, we expect the boundary brane to be the familiar D6-brane moving in D9-brane, alias the ten dimensional spacetime. Therefore let us concentrate on the remaining cases of $\mathrm{D} p$-branes ending on $\mathrm{D}(p+2)$-branes with $1 \leq p \leq 6$. All of these are codimension 3 embeddings in which the boundary $\mathrm{D}(p-1)$-brane is propagating in $(p+3)$ dimensions. The $3,4,5$ branes propagating in 7,8,9 dimensional target spacetimes, resepectively, have already been encountered in the context of superembeddings in [30], where they were called the L-branes. It was argued in [30] that the associated supermebedding constraints imply the equations appropriate to linear multiplets which happen to be off-shell supermultiplets.

Consider the L5-brane in 9 dimensions (the description of the other cases can be obtained by dimensional reduction). Its worldvolume multiplet consists of three scalars, a 4-form potential and a spinor with 8 real components. So the off-shell degrees of freedom count is $3+5$ bosons and 8 fermions. Interestingly, there are no auxiliary fields in this multiplet. The existence of 4 -form potentials on the worldvolume leads, by arguments similar to those of the previous section, to the boundary $\mathcal{F}$-constraint [31]

$$
d \mathcal{F}_{5}=\left(f_{5}^{8}\right)^{*} G_{6}
$$

where $G_{6}$ is a closed super-form in 9 dimensions. However, the system remains off-shell even in presence of this constraint. In order to put the system on-shell, one has to construct an action that yields the equations of motions. We will show elsewhere that this is indeed possible [31].

In passing we note that putting the linear multiplet on-shell means that the 4 -form potential obeys a Maxwell type equation and therefore on-shell it is dual to a scalar field. Since the fermions describe 4 degrees of freedom, one then obtains a $4+4$ on-shell multiplet which is essentially a hypermultiplet with one of the scalars dualized to a 4 -form potential. This system is therefore intimately related to a vertical reduction of a 5-brane in 10 dimensions, followed by the dualization of the 10th coordinate scalar to a 4 -form potential on the 5-brane worldvolume 30].

In this paper we focused on M2-brane ending on M5-brane, $\mathrm{D} p$-branes ending on $\mathrm{D}(p+2)$-branes and fundamental string ending on $\mathrm{D} p$-branes. In the firt stwo cases we assumed that the open branes have single component boundaries, while in the last case we let the two ends of the open string lie on a single D $p$-brane, for simplicity. Not all of these configurations are necessarily BPS saturated or anomaly free. While anomaly freedom is essential, the BPS saturation is less crucial property since the BPS states presumably constitute only a tiny fraction of all possible states.

The universal nature of the superembedding formalism suggests that it can successfully be applied to many other generalizations of the systems studied in this paper. For example, it can be applied straightforwardly to D $p$-branes ending on NS 5-branes. One can also treat configurations in which the open M2-brane ends on an M5-brane at one end and an M9-brane on the other, or both ends ending on M9-branes (when we discuss M9-branes, we have in mind the Horawa-Witten picture of such objects as boundaries of the eleven dimensional spacetime 
with suitable topology [2]).

One may also consider a system in which the open M2-brane has multi-component boundaries which may end on any M5-branes or M9-branes in all possible ways. It is clear that there is a rich spectrum of possibilities due to the fact that the basic building blocks can have a large class of nontrivial topologies. Further novel possibilities can also arise because brane theories are intrinsically nonlinear and consequently the topology of branes can change through selfinteractions.

\section{Acknowledments}

It is a pleasure to thank M. Duff and P. Sundell for stimulating discussions.

\section{References}

[1] E. Bergshoeff, E. Sezgin and P.K. Townsend, Properties of the eleven dimensional supermembrane theory, Annals Phys. 185330.

[2] P. Horava and E. Witten, Heterotic and type I string dynamics from eleven dimensions, Nucl. Phys. B460 (1996) 506, hep-th/9510209.

[3] A. Strominger, Open p-branes, Phys. Lett. B383 (1996) 44, hep-th/9512059.

[4] P.K. Townsend, D-branes from M-branes, Phys. Lett. B373 (1996) 68, hep-th/9512062.

[5] K. Becker and M. Becker, Boundaries in M-theory, Nucl.Phys. B472 (1996) 221, hepth/9602071.

[6] F. Aldabe, Heterotic and Type I strings from twisted supermembranes, Nucl. Phys. B473 (1996) 63, hep-th/9603183.

[7] P.K. Townsend, Brane surgery, Nucl. Phys. Proc. Suppl. 58 (1997) 163, hep-th/9609217.

[8] M. Cederwall, Boundaries of 11-dimensional membranes, Mod. Phys. Lett. A12 (1997) 2641, hep-th/9704161.

[9] Ph. Brax and J. Mourad, Open membranes in eleven dimensions, Phys. Lett. B408 (1997) 142, hep-th/9704165.

[10] K. Ezawa, Y. Matsuo and K. Murakami, Matrix regularization of open supermembrane towards M-theory five-brane via open supermembrane, hep-th/9707200.

[11] Ph. Brax and J. Mourad, Open supermembrane coupled to $M$ - theory five-branes, hepth/9707246.

[12] B. de Wit, K. Peeters and J. Plefka, Open and closed supermembranes with winding, hepth/9710215.

[13] R. Argurio, F. Englert, L. Houart and P. Windey, On the opening of branes, Phys. Lett. B408 (1997) 151, hep-th/9704190.

[14] E. Bergshoeff, J. Gomis, P.K. Townsend, M-brane intersections from worldvolume superalgebras, hep-th/9711043. 
[15] C.S. Chu and E. Sezgin, M-Fivebrane from the open supermembrane, JHEP 12 (1997) 001, hep-th/9710223.

[16] C.S. Chu, P.S. Howe and E. Sezgin, Strings and D-branes with boundaries, hep-th/9801202.

[17] J. Schwarz, Lectures on Superstrings and M theory dualities, hep-th/9607201.

[18] O. Aharony, J. Sonnenschein and S. Yankielowicz, Interactions of strings and D-branes from $M$ theory, Nucl. Phys. B474 (1996) 309, hep-th/9603009.

[19] A. Sen, String network, hep-th/9711130.

[20] O. Aharony, A. Hanany, B. Kol, Webs of $(p, q)$ 5-branes, five dimensional field theories and grid diagrams, hep-th/9710116.

[21] P.S. Howe, N.D. Lambert and P.C. West, The self-dual string soliton, hep-th/9709014.

[22] P.S. Howe and E. Sezgin, $D=11, p=5$, Phys. Lett. B394 (1997) 62, hep-th/9611008.

[23] P.S. Howe, E. Sezgin and P.C. West, Covariant field equations of the M-theory five-brane, Phys. Lett. 399B (1997) 49, hep-th/9702008.

[24] P. Pasti and M. Tonin, Twistor-like formulation of the supermembrane in $D=11$, Nucl. Phys. B418 (1994) 337, hep-th/9303156.

[25] E. Bergshoeff and E. Sezgin, Twistor-like formulation of super p-branes, Nucl. Phys. B422 (1994) 329, hep-th/9312168.

[26] P.S. Howe, O. Raetzel and E. Sezgin, On brane actions and superembeddings, in preparation.

[27] P.S. Howe, O. Raetzel, E. Sezgin and P. Sundell, D-brane superembeddings, in preparation.

[28] V. Akulov, I. Bandos, W. Kummer and V. Zima, D=10 Dicichlet super-9-brane, hepth/9802032.

[29] E. Bergshoeff and P.K. Townsend, Super D-branes, Nucl. Phys. B490 (1997) 145, hepth/9611173.

[30] P.S. Howe and E. Sezgin, Superbranes, Phys. Lett. B390 (1997) 133, hep-th/9607227.

[31] P.S. Howe, O. Raetzel, I. Rudychev and E. Sezgin, L-branes, in preparation. 